\title{
Heat Transfer due to Magnetohydrodynamic Stagnation-Point Flow of a Power-Law Fluid towards a Stretching Surface in the Presence of Thermal Radiation and Suction/Injection
}

\author{
Tapas Ray Mahapatra, ${ }^{1}$ Sabyasachi Mondal, ${ }^{2}$ and Dulal Pal ${ }^{1}$ \\ ${ }^{1}$ Department of Mathematics, Visva Bharati (A Central University), West Bengal, Santiniketan 731235, India \\ ${ }^{2}$ Department of Mathematics, Bengal Institute of Technology and Management, West Bengal, Santiniketan 731236, India \\ Correspondence should be addressed to Sabyasachi Mondal, sabya.mondal.2007@gmail.com
}

Received 5 October 2012; Accepted 19 November 2012

Academic Editors: A. Ghoufi, S. Hashimoto, and B. Merinov

Copyright ( $\odot 2012$ Tapas Ray Mahapatra et al. This is an open access article distributed under the Creative Commons Attribution License, which permits unrestricted use, distribution, and reproduction in any medium, provided the original work is properly cited.

\begin{abstract}
An analysis is made on the study of two-dimensional MHD (magnetohydrodynamic) boundary-layer stagnation-point flow of an electrically conducting power-law fluid over a stretching surface when the surface is stretched in its own plane with a velocity proportional to the distance from the stagnation-point in the presence of thermal radiation and suction/injection. The paper examines heat transfer in the stagnation-point flow of a power-law fluid except when the ratio of the free stream velocity and stretching velocity is equal to unity. The governing partial differential equations along with the boundary conditions are first brought into a dimensionless form and then the equations are solved by Runge-Kutta fourth-order scheme with shooting techniques. It is found that the temperature at a point decreases/increases with increase in the magnetic field when free stream velocity is greater/less than the stretching velocity. It is further observed that for a given value of the magnetic parameter $M$, the dimensionless rate of heat transfer at the surface and $\left|\theta^{\prime}(0)\right|$ decreases/increases with increase in the power-law index $n$. Further, the temperature at a point in the fluid decreases with increase in the radiation parameter $N_{R}$ when free stream velocity is greater/less than the stretching velocity.
\end{abstract}

\section{Introduction}

Recently, a considerable amount of interest has been evinced in the study of MHD (magnetohydrodynamic) stagnationpoint flow and heat transfer of a power-law fluid towards a stretching surface in view of its various industrial applications such as extrusion of polymer sheets, continuous stretching, manufacturing plastic films, and artificial fibres. In a melt-spinning process, the extrudate from the die is usually drawn and simultaneously stretched into a sheet which is then solidified through quenching or gradual cooling by direct contact with water. The qualities of the final product depend on the rate of heat transfer at the stretching surface. It is, therefore, of great importance to know the flow behavior over a stretching surface which determines the rate of cooling.
The results of these studies are of great technical importance; for example, in the prediction of skin-friction, as well as heat/mass transfer near stagnation regions of bodies in high speed flows. Sapunkov [1] investigated the two-dimensional orthogonal stagnation-point flow of an incompressible electrically conducting power-law fluid towards a rigid surface in the presence of a uniform transverse magnetic field. Crane [2] studied the steady flow of an incompressible viscous fluid over a deformable sheet, the flow being caused solely by the stretching of the sheet in its own plane with a velocity varying linearly with the distance from a fixed point. Pavlov [3] gave an exact similarity solution to the MHD boundary layer equations for the steady two-dimensional flow of an electrically conducting incompressible fluid due to the stretching of a plane elastic surface in the presence of a uniform transverse magnetic field. A comprehensible discussion on the potential 
engineering application of non-Newtonian power-law electrically conducting fluids permeated by magnetic fields was presented by Martinson and Pavlov [4]. Heat transfer in the flow over a stretching surface maintained at constant as well as variable temperature was analyzed by P. S. Gupta and A. S. Gupta [5] and Carragher and Crane [6]. Massoudi and Ramezan [7] used a perturbation technique to study the stagnation point flow and heat transfer of a second grade non-Newtonian fluid. Their analysis is valid only for small values of the parameter that determines the behavior of the non-Newtonian fluid. Later, Massoudi and Ramezan [8] extended the problem to nonisothermal surfaces. MHD flow of a power-law fluid over a stretching surface was examined by Andersson et al. [9]. Garg [10] improved the solution obtained by Massoudi and Ramezan [7] by numerically computing the flow characteristics for any value of the nonNewtonian parameter using a pseudo-similarity solution. Mahapatra and Gupta [11] analyzed the steady twodimensional orthogonal stagnation-point flow of an incompressible viscous electrically conducting fluid towards a stretching surface. Steady two-dimensional orthogonal stagnation-point flow of an incompressible viscous fluid towards a stretching surface was studied by Mahapatra and Gupta [12] who also investigated heat transfer in this flow. Many authors presented some mathematical results on flow and heat transfer and a good amount of references can be found in the papers by Liao and Pop [13] and Nazar et al. [14]. Prasad et al. [15] studied MHD power-law fluid flow and heat transfer over a non-isothermal stretching sheet. Recently, Mahapatra et al. [16] studied the steady two-dimensional orthogonal stagnation-point flow of an electrically conducting power-law fluid towards a stretching surface when the surface is stretched in its own plane with velocity proportional to the distance from the stagnation-point.

A new dimension is added to the study of flow and heat transfer over a stretching sheet by consisting the effect of thermal radiation. It is well known that the effect of thermal radiation is important in space technology and high temperature processes. Thermal radiation also plays an important role in controlling heat transfer process in polymer processing industry. The effect of radiation on heat transfer problems has been studied by Hossain and Takhar [17]. Recently, Pal [18] has investigated heat and mass transfer in stagnation-point flow towards a stretching surface in the presence of buoyancy force and thermal radiation. The objective of this paper is to study the heat transfer in steady two-dimensional orthogonal stagnation-point flow of an electrically conducting power-law fluid towards a stretching surface in the presence of a magnetic field and thermal radiation. Temperature of the wall and temperature of free stream fluid are kept constant in such a way that the former is higher than the later.

\section{Flow Analysis}

Consider two-dimensional steady stagnation-point flow of an electrically conducting power-law fluid towards a flat surface coinciding with the plane $y=0$ in the presence of a uniform transverse magnetic field $B_{0}$, the flow being

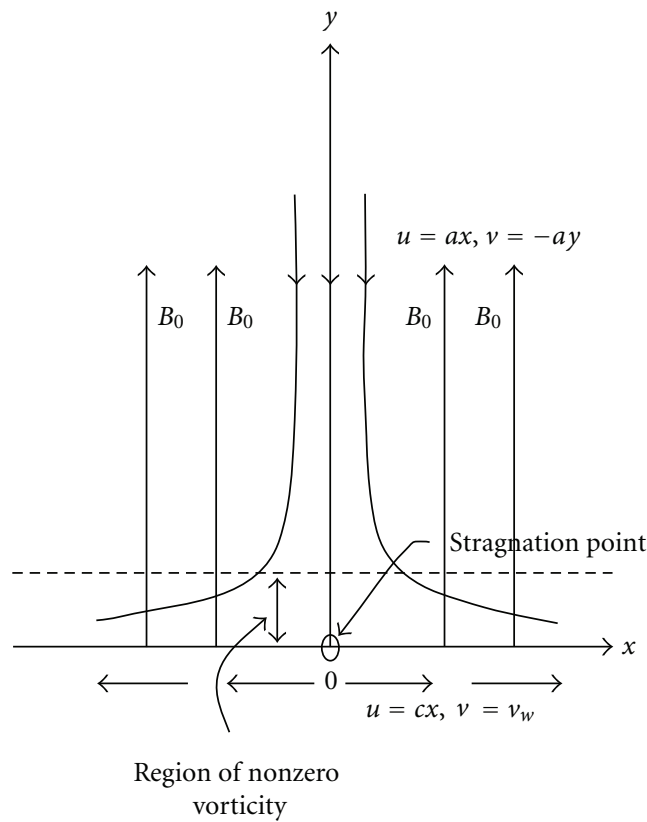

FIGURE 1: A sketch of the physical problem.

confined to the region $y>0$. Two equal and opposite forces are applied on the stretching surface along the $x$-axis so that the surface is stretched keeping the origin fixed as shown in Figure 1. The MHD equations for steady two-dimensional stagnation-point flow in the boundary layer towards the stretching surface are as follows:

$$
\begin{gathered}
\frac{\partial u}{\partial x}+\frac{\partial v}{\partial y}=0 \\
u \frac{\partial u}{\partial x}+v \frac{\partial u}{\partial y}=U \frac{\partial U}{\partial x}+\frac{1}{\rho} \frac{\partial \tau_{x y}}{\partial y}-\frac{\sigma B_{0}^{2}}{\rho}(u-U)
\end{gathered}
$$

where the induced magnetic field is neglected (which is justified for MHD flow at small magnetic Reynolds number by Shercliff [19]). It is also assumed that the external electric field is zero and the electric field due to polarization of charges is negligible. Here $u$ and $v$ are the velocity components along the $x$ and $y$ directions, respectively. Further $\rho, \sigma$, $B_{0}$, and $\tau_{x y}$ denote density of the fluid, electrical conductivity, applied magnetic field, and shear stress, respectively. In (2), $U(x)$ stands for the stagnation-point velocity in the inviscid free stream. Here the stress tensor is defined by (Mahapata and Gupta [12])

$$
\tau_{i j}=2 K\left|2 D_{i j} D_{i j}\right|^{(n-1) / 2} D_{i j},
$$

where

$$
D_{i j}=\frac{1}{2}\left(\frac{\partial u_{i}}{\partial x_{j}}+\frac{\partial u_{j}}{\partial x_{i}}\right)
$$

denotes the rate of strain tensor, $K$ is the consistency coefficient and $n$ is the power-law index. Fluids obeying constitutive equation (3) are called power-law fluid. It is called 
pseudoplastic power-law fluid when $n<1$ and dilatant power-law fluid when $n>1$ since the apparent viscosity decreases (shear-thinning) or increases with the increase in shear rate (shear-thickening) accordingly as $n<1$ or $n>1$. It is called Newtonian fluid when $n=1$. Here we denote $u_{1}=u$ and $u_{2}=v$. In the present problem, we have $\partial u / \partial y<0$ when $a / c<1$ and $\partial u / \partial y>0$ when $a / c>1$, which gives the shear stress as

$$
\begin{gathered}
\tau_{x y}=-K\left(-\frac{\partial u}{\partial y}\right)^{n} \quad \text { when } \frac{a}{c}<1 \\
\tau_{x y}=K\left(\frac{\partial u}{\partial y}\right)^{n} \quad \text { when } \frac{a}{c}>1 .
\end{gathered}
$$

When $a / c<1$, the momentum equation (2) becomes

$$
u \frac{\partial u}{\partial x}+v \frac{\partial u}{\partial y}=U \frac{\partial U}{\partial x}-\frac{K}{\rho} \frac{\partial}{\partial y}\left(-\frac{\partial u}{\partial y}\right)^{n}-\frac{\sigma B_{0}^{2}}{\rho}(u-U),
$$

and when $a / c>1$, (2) becomes

$$
u \frac{\partial u}{\partial x}+v \frac{\partial u}{\partial y}=U \frac{\partial U}{\partial x}+\frac{K}{\rho} \frac{\partial}{\partial y}\left(\frac{\partial u}{\partial y}\right)^{n}-\frac{\sigma B_{0}^{2}}{\rho}(u-U) .
$$

Here $B_{0}$ is the externally imposed magnetic field in the $y$ direction. The appropriate boundary conditions are

$$
\begin{gathered}
u=c x, \quad v=v_{w}(x) \quad \text { at } y=0, \\
u \longrightarrow U(x)=a x, \quad v=-a y \quad \text { as } y \longrightarrow \infty,
\end{gathered}
$$

where $a$ and $c$ are positive constants and $v_{w}(x)=s x^{(n-1) /(n+1)}$, where $s$ is constant.

We introduce the variables as

$$
\begin{gathered}
\psi=\left(\frac{K / \rho}{c^{1-2 n}}\right)^{1 /(n+1)} x^{2 n /(n+1)} F(\eta), \\
\eta=y\left(\frac{c^{2-n}}{K / \rho}\right)^{1 /(n+1)} x^{(1-n) /(1+n)},
\end{gathered}
$$

where stream function $\psi$ is defined by the following relations

$$
u=\frac{\partial \psi}{\partial y}, \quad v=-\frac{\partial \psi}{\partial x} .
$$

Using (9), (6) and (7) now become, when $a / c<1$ as

$$
\begin{gathered}
n\left[-F^{\prime \prime}(\eta)\right]^{(n-1)} F^{\prime \prime \prime}(\eta)+\left(\frac{2 n}{n+1}\right) F(\eta) F^{\prime \prime}(\eta) \\
-F^{\prime 2}(\eta)-M F^{\prime}(\eta)+M \frac{a}{c}+\frac{a^{2}}{c^{2}}=0
\end{gathered}
$$

and when $a / c>1$, we have

$$
\begin{gathered}
n\left[F^{\prime \prime}(\eta)\right]^{(n-1)} F^{\prime \prime \prime}(\eta)+\left(\frac{2 n}{n+1}\right) F(\eta) F^{\prime \prime}(\eta) \\
-F^{\prime 2}(\eta)-M F^{\prime}(\eta)+M \frac{a}{c}+\frac{a^{2}}{c^{2}}=0 .
\end{gathered}
$$

From (8), we get the dimensionless boundary conditions as

$$
F(0)=f_{w}, \quad F^{\prime}(0)=1, \quad F^{\prime}(\infty)=\frac{a}{c},
$$

where the prime denotes differentiation with respect to $\eta$.

Here $f_{w}=-\left[(s((n+1) / 2 n)) /\left((K / \rho) / c^{1-2 n}\right)^{1 /(n+1)}\right]$ that is, $f_{w}<0$ injection, $f_{w}>0$ suction and $f_{w}=0$ absence of suction/injection parameter and $M\left(=\sigma B_{0}^{2} / \rho c\right)$ is the magnetic parameter. The skin friction coefficient $C_{f}$ at the wall is given by

$$
\begin{aligned}
C_{f} & =\frac{\tau_{w}}{(1 / 2) \rho(c x)^{2}}=2\left[-F^{\prime \prime}(0)\right]^{n}\left[\frac{(c x)^{2-n} x^{n}}{K / \rho}\right]^{-1 /(1+n)} \\
& =2\left[-F^{\prime \prime}(0)\right]^{n}\left(\operatorname{Re}_{x}\right)^{-1 /(1+n)} \quad \text { when } \frac{a}{c}<1, \\
C_{f} & =\frac{\tau_{w}}{(1 / 2) \rho(c x)^{2}}=2\left[F^{\prime \prime}(0)\right]^{n}\left[\frac{(c x)^{2-n} x^{n}}{K / \rho}\right]^{-1 /(1+n)} \\
& =2\left[F^{\prime \prime}(0)\right]^{n}\left(\operatorname{Re}_{x}\right)^{-1 /(1+n)} \quad \text { when } \frac{a}{c}>1,
\end{aligned}
$$

where $\operatorname{Re}_{x}=(c x)^{2-n} x^{n} /(K / \rho)$ is local Reynolds number based on the sheet velocity $c x$. The equations (11) and (12) are solved numerically as described in Mahapatra et al. [16] for different values of power-law index $n$ in the range $0.4 \leq$ $n \leq 2.0$ and for different values of $a / c$ and $f_{w}$.

\section{Heat Transfer}

Let us consider the heat transfer equation in the stagnationpoint flow of electrically conducting power-law fluid towards a stretching surface. Using boundary layer approximations and neglecting viscous and Ohmic dissipation, the transfer of heat in the steady two-dimensional flow of a power-law fluid can be expressed in the form of the energy equation in the presence of thermal radiation is given by

$$
u \frac{\partial T}{\partial x}+v \frac{\partial T}{\partial y}=\lambda \frac{\partial^{2} T}{\partial y^{2}}-\frac{1}{\rho C_{p}} \frac{\partial q_{r}}{\partial y},
$$

where $T, \lambda, q_{r}$, and $C_{p}$ denote temperature, thermal diffusivity, the radiation heat flux, and specific heat at constant pressure of the fluid. In addition, the radiation heat flux $q_{r}$ is considered according to Rosseland approximation such that

$$
q_{r}=-\frac{4 \sigma^{*}}{3 K^{*}} \frac{\partial T^{4}}{\partial y},
$$

where $\sigma^{*}$ and $K^{*}$ are the Stefan-Boltzmann constant and the mean absorption coefficient, respectively. As done by Raptis [20], the fluid-phase temperature differences within the flow are assumed to be sufficiently small so that $T^{4}$ may be expressed as a linear function of temperature. This is done by expanding $T^{4}$ in a Taylor series about the free stream temperature $T_{\infty}$ and neglecting higher order terms to yield,

$$
T^{4}=4 T_{\infty}^{3} T-3 T_{\infty}^{4} .
$$


TABLE 1: Comparison values of $-F^{\prime \prime}(0)$ for various values of $a / c, n$ and $M$ when $f_{w}=0$ with Mahapatra et al. [16].

\begin{tabular}{|c|c|c|c|c|c|c|c|}
\hline \multirow{2}{*}{$a / c$} & \multirow{2}{*}{$n$} & \multicolumn{3}{|c|}{ Mahapatra et al. [16] } & \multicolumn{3}{|c|}{ Present work } \\
\hline & & $M \rightarrow 0.0$ & 0.5 & 1.0 & $M \rightarrow 0.0$ & 0.5 & 1.0 \\
\hline \multirow{5}{*}{0.2} & 0.4 & 1.1593 & 1.5176 & 1.8435 & 1.1593 & 1.5176 & 1.8435 \\
\hline & 0.6 & 1.0018 & 1.2506 & 1.4725 & 1.0018 & 1.2506 & 1.4725 \\
\hline & 0.8 & 0.9424 & 1.1356 & 1.3058 & 0.9424 & 1.1356 & 1.3058 \\
\hline & 1.0 & 0.9181 & 1.0768 & 1.2156 & 0.9181 & 1.0768 & 1.2156 \\
\hline & 1.2 & 0.9087 & 1.0437 & 1.1612 & 0.9087 & 1.0437 & 1.1612 \\
\hline \multirow{5}{*}{2.0} & 0.4 & -3.7731 & -4.1322 & -4.4796 & -3.7731 & -4.1322 & -4.4796 \\
\hline & 0.6 & -2.7631 & -2.9827 & -3.1933 & -2.7631 & -2.9827 & -3.1933 \\
\hline & 0.8 & -2.2893 & -2.4445 & -2.5925 & -2.2893 & -2.4445 & -2.5925 \\
\hline & 1.0 & -2.0175 & -2.1363 & -2.2491 & -2.0175 & -2.1363 & -2.2491 \\
\hline & 1.2 & -1.8421 & -1.9377 & -2.0282 & -1.8421 & -1.9377 & -2.0282 \\
\hline
\end{tabular}

Using (16) and (17), we obtain from (15)

$$
u \frac{\partial T}{\partial x}+v \frac{\partial T}{\partial y}=\lambda \frac{\partial^{2} T}{\partial y^{2}}+\frac{16 \sigma^{*}}{3 K^{*}} \frac{T_{\infty}^{3}}{\rho C_{p}} \frac{\partial^{2} T}{\partial y^{2}} .
$$

The appropriate boundary conditions are

$$
\begin{gathered}
T=T_{w} \text { at } y=0, \\
T \longrightarrow T_{\infty} \text { as } y \longrightarrow \infty,
\end{gathered}
$$

where $T_{w}$ and $T_{\infty}$ are constants with $T_{w}>T_{\infty}$. Now the energy equation (18), with the above boundary condition, becomes

$$
\operatorname{Pr}^{-1}\left(3 N_{R}+4\right) \theta^{\prime \prime}(\eta)+\frac{6 n N_{R}}{(n+1)} F(\eta) \theta^{\prime}(\eta)=0,
$$

where

$$
\theta(\eta)=\frac{T(x, y)-T_{\infty}}{T_{w}-T_{\infty}}
$$

subject to

$$
\theta(0)=1, \quad \theta(\infty)=0
$$

Here $N_{R}=\lambda K^{*} \rho C_{p} / 4 \sigma^{*} T_{\infty}^{3}$ and $\operatorname{Pr}=(c / \lambda)((K / \rho) /$ $\left.c^{2-n}\right) \operatorname{Re}_{x}^{(n-1) /(n+1)}$ are the radiation parameter and the local Prandtl number, respectively. The Equations (20) and (22) are solved numerically using Runge-Kuttta fourth-order method with shooting technique.

\section{Discussion of the Results}

The nonlinear boundary value problem (11), (12), and (20) cannot be solved in closed form, so these equations are solved numerically using fourth-order Runge-Kutta method with shooting technique. Extensive calculations have been performed to obtain the flow and temperature profiles for various values of physical parameters such as $n, M, N_{R}$, Pr, and so forth as taken by Mahapatra and Gupta [11], Mahapatra et al. [16]. We have compared numerical result for $F^{\prime \prime}(0)$ with those obtained by Mahapatra et al. [16] in
Table 1 for various values of $a / c, n$, and $M$ in absence of suction/injection. Table 2 shows the computed values of $-F^{\prime \prime}(0)$ for various values of $a / c, n$, and $M$ when $f_{w}=-1$ and $f_{w}=1$. From this table, it is seen that $F^{\prime \prime}(0)$ is negative when $a / c<1$ and $F^{\prime \prime}(0)$ is positive when $a / c>1$. It is also seen that $\left|F^{\prime \prime}(0)\right|$ decreases with increase in $n$ for fixed $M$ for both $a / c<1$ and $a / c>1$. It is also observed that $\left|F^{\prime \prime}(0)\right|$ increases with increase in $M$ for fixed $n$ for both $a / c<1$ and $a / c>1$. The computed values of $-\theta^{\prime}(0)$ for various values of $a / c, n$, and $M$ with $N_{R}=1.0, \operatorname{Pr}=2.0$ when $f_{w}=-1,0,1$ are presented in Table 3. From this table, it is seen that $\left|\theta^{\prime}(0)\right|$ increases with increasing $n$ when $a / c>1$ for fixed values of $N_{R}, \operatorname{Pr}$, and $M$ for $f_{w}=-1,0,1$. The same trend is observed by increasing the value of $n$ when $a / c<1$ for $f_{w}=0,1$ and opposite trend is observed when for $f_{w}=-1$. It is also seen that $\left|\theta^{\prime}(0)\right|$ increases with increasing $M$ when $a / c>1$ for fixed values of $n, \operatorname{Pr}$ and $N_{R}$ when $f_{w}=-1,0,1$ and opposite trend is seen for $a / c<1$ when $f_{w}=-1,0,1$.

Figure 2 shows the variation of $x$-component of velocity for different values of $f_{w}$ and $a / c$ for fixed values of $M=1.0$ and $n=0.2$ and 2.0. It is clear from this figure that when $a / c<1$, the velocity at a point in the neighbourhood of stretching plate decreases with the increase in the suction parameter $f_{w}$, whereas when $a / c>1$ the opposite trend is observed. In both cases velocity at infinity coincides with the values of $a / c$ for all values of $f_{w}$ which is also seen from this figure. Figure 3 shows that the temperature at a given point decreases with increase in $f_{w}$ for both the cases when $a / c<1$ and $a / c>1$ for fixed values of $M, n, \operatorname{Pr}$, and $N_{R}$. Further, this figure shows that the temperature at a given point decreases with increasing $a / c$ for fixed values of $M, n, \operatorname{Pr}$, and $N_{R}$.

Figure 4 shows the variation of $x$-component velocity profile for different values of $n$ and $a / c$ for fixed values of $M$. It is clear from this figure that when $a / c<1$, the velocity at a point in a neighbourhood of the stretching plate increases with increase in $n$. When $a / c>1$ the opposite trend is observed. In both cases velocity at infinity coincides with the values of $a / c$ for all $n$ which is also seen from this figure. It can also be seen that when $a / c>1$, the flow has a boundary layer structure and the thickness of this boundary layer structure increases with increase in $n$. It is interesting to note that when $a / c<1$, the flow has an inverted boundary layer structure. 
TABLE 2: Computed values of $-F^{\prime \prime}(0)$ for various values of $a / c, n$ and $M$ when $f_{w}=-1$ and $f_{w}=1$.

\begin{tabular}{|c|c|c|c|c|c|c|c|}
\hline \multirow{2}{*}{$a / c$} & \multirow{2}{*}{$n$} & \multicolumn{3}{|c|}{$f_{w}=-1$} & \multicolumn{3}{|c|}{$f_{w}=1$} \\
\hline & & $M \rightarrow 0.0$ & 0.5 & 1.0 & $M \rightarrow 0.0$ & 0.5 & 1.0 \\
\hline \multirow{4}{*}{0.2} & 0.4 & 0.7889 & 1.0627 & 1.3188 & 1.8020 & 2.2688 & 2.6810 \\
\hline & 0.6 & 0.6661 & 0.8698 & 1.0560 & 1.5622 & 1.8501 & 2.1023 \\
\hline & 0.8 & 0.6135 & 0.7837 & 0.9364 & 1.4651 & 1.6628 & 1.8375 \\
\hline & 1.0 & 0.5869 & 0.7381 & 0.8713 & 1.4130 & 1.5579 & 1.6873 \\
\hline \multirow{4}{*}{2.0} & 0.4 & -2.6414 & -2.9383 & -3.2269 & -5.5392 & -5.9672 & -6.3796 \\
\hline & 0.6 & -1.9991 & -2.1967 & -2.3866 & -3.8431 & -4.0789 & -4.3050 \\
\hline & 0.8 & -1.6947 & -1.8443 & -1.9868 & -3.0686 & -3.2228 & -3.3070 \\
\hline & 1.0 & -1.5203 & -1.6418 & -1.7567 & -2.6293 & -2.74038 & -2.8466 \\
\hline
\end{tabular}

TABLe 3: Computed values of $-\theta^{\prime}(0)$ for various values of $a / c, n$ and $M$ with $N_{R}=1.0, \operatorname{Pr}=2.0$ when $f_{w}=-1,0,1$.

\begin{tabular}{ccccccccccc}
\hline$a / c$ & $n$ & & \multicolumn{3}{c}{$f_{w}=-1$} & & $f_{w}=0$ & & \multicolumn{3}{c}{$f_{w}=1$} \\
& & $M \rightarrow 0.0$ & 0.5 & 1.0 & $M \rightarrow 0.0$ & 0.5 & 1.0 & $M \rightarrow 0.0$ & 0.5 \\
\hline \multirow{4}{*}{0.2} & 0.4 & 0.1668 & 0.1435 & 0.1263 & 0.4159 & 0.3919 & 0.3753 & 0.7664 & 0.7489 & 0.7361 \\
& 0.6 & 0.1659 & 0.1420 & 0.1250 & 0.4885 & 0.4659 & 0.4492 & 0.9538 & 0.9392 & 0.9282 \\
& 0.8 & 0.1633 & 0.1493 & 0.1243 & 0.5358 & 0.5156 & 0.5023 & 1.0926 & 1.0809 & 1.0717 \\
& 1.0 & 0.1583 & 0.1364 & 0.1205 & 0.5684 & 0.5504 & 0.5434 & 1.2006 & 1.1914 & 1.1838 \\
\hline \multirow{4}{*}{2.0} & 0.4 & 0.4376 & 0.4413 & 0.4445 & 0.7314 & 0.7347 & 0.7276 & 1.0636 & 1.0662 & 1.0685 \\
& 0.6 & 0.4459 & 0.4540 & 0.4536 & 0.8092 & 0.8128 & 0.8160 & 1.2617 & 1.2645 & 1.2670 \\
& 0.8 & 0.4461 & 0.4503 & 0.4541 & 0.8685 & 0.8722 & 0.8754 & 1.4072 & 1.4099 & 1.4124 \\
& 1.0 & 0.4489 & 0.4510 & 0.4556 & 0.9120 & 0.9155 & 0.9187 & 1.5197 & 1.5221 & 1.5245 \\
\hline
\end{tabular}

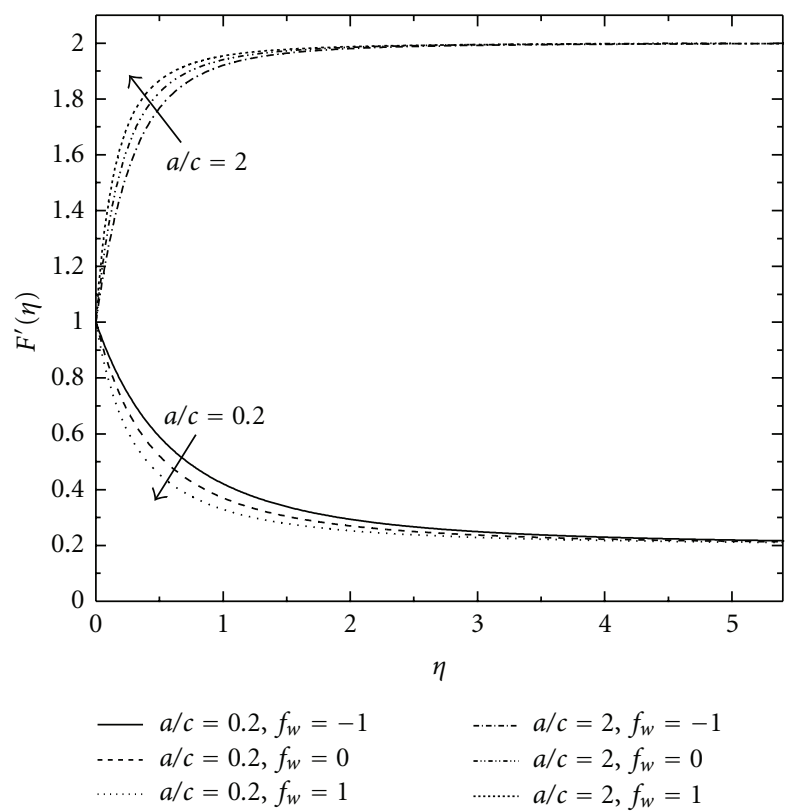

FIGURE 2: Variation of $F^{\prime}(\eta)$ with $\eta$ for several values of $f_{w}$ and $a / c$ when $M=1.0, n=0.4, N_{R}=1.0$ and $\operatorname{Pr}=2.0$.

This is due to the fact when $a / c<1$, the stretching velocity of the surface $(c x)$ exceeds the velocity of the external stream (ax).

Figures 5, 6, and 7 demonstrate that the temperature at a given point decreases with increasing $\operatorname{Pr}$ for both $a / c<1$

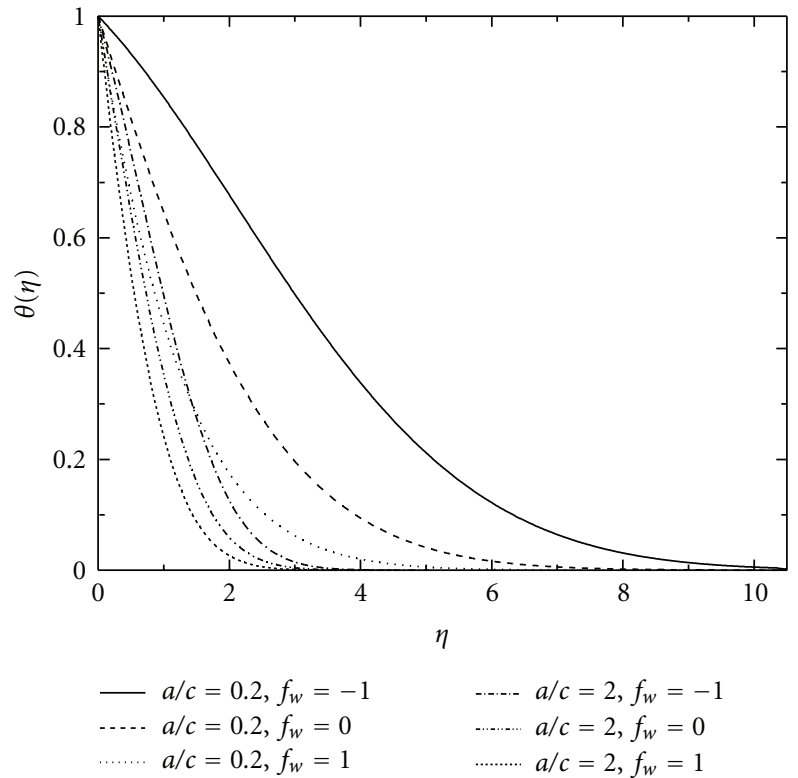

Figure 3: Variation of $\theta(\eta)$ with $\eta$ for different values of $f_{w}$ and $a / c$ with $M=1.0, n=0.4, N_{R}=1.0$ and $\operatorname{Pr}=2.0$.

and $a / c>1$, this is due to the fact that the thermal boundary layer thickness decreases with increase in the value of Prandtl number for fixed values of $n, M$, and $N_{R}$ when $f_{w}=-1,0,1$. Figures 8,9 , and 10 show the variation of temperature profiles for various values of $a / c$ and $n$ in the thermal boundary 


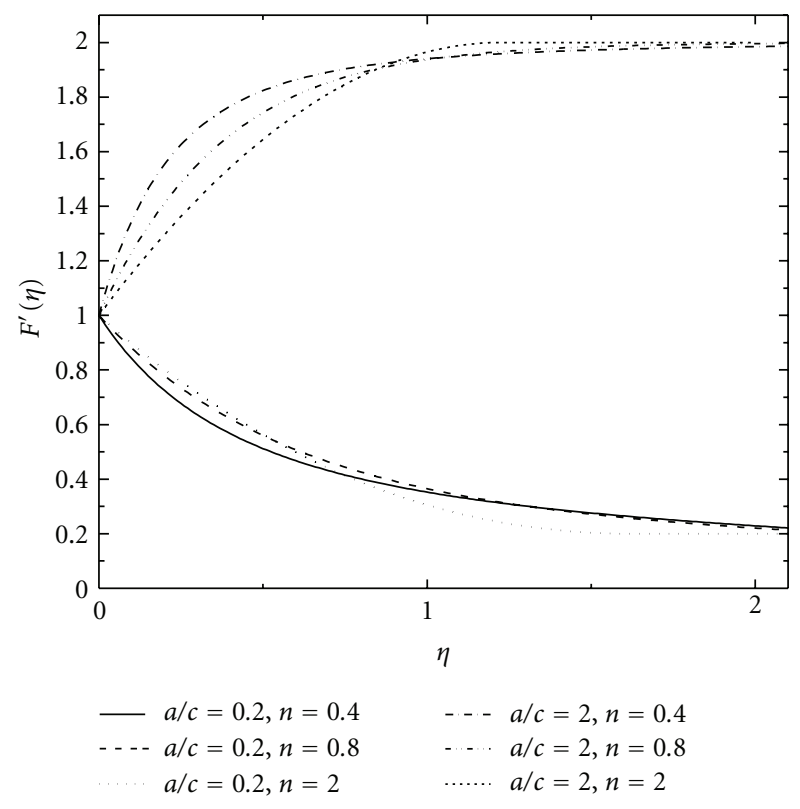

FIGURE 4: Variation of $F^{\prime}(\eta)$ with $\eta$ for several values of $n$ and $a / c$ when $f_{w}=0, M=1.0$.

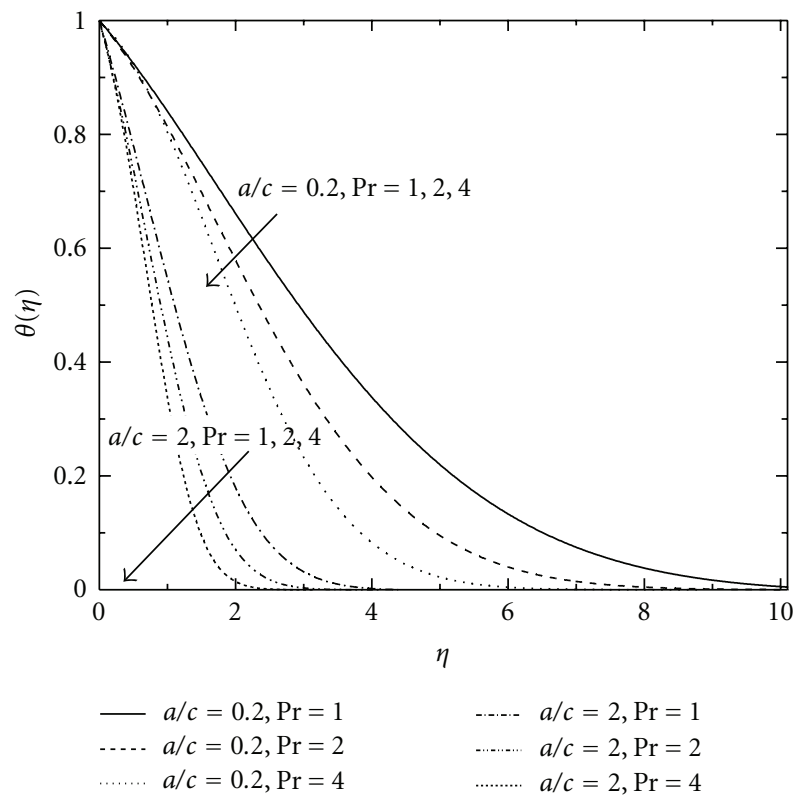

Figure 5: Variation of $\theta(\eta)$ with $\eta$ for different values of $\operatorname{Pr}$ and $a / c$ with $f_{w}=-1, n=0.8, M=0.5$, and $N_{R}=1.0$.

layer for fixed values of $M, N_{R}$, and $\operatorname{Pr}$ when $f_{w}=-1,0,1$, respectively. From this figures, it is observed that the temperature profiles decreases with increasing $n$ for both $a / c<1$ and $a / c>1$. Further, these figures show that the temperature at a given point decreases with increasing $a / c$ for fixed values of $M, n, \operatorname{Pr}$, and $N_{R}$. Figures 11,12 , and 13 reveal that the temperature at a given point decreases with increasing radiation parameter $N_{R}$ for both $a / c<1$ and $a / c>1$ for fixed values of $M, \operatorname{Pr}$, and $n$ when $f_{w}=-1,0,1$. Due to the fact that the

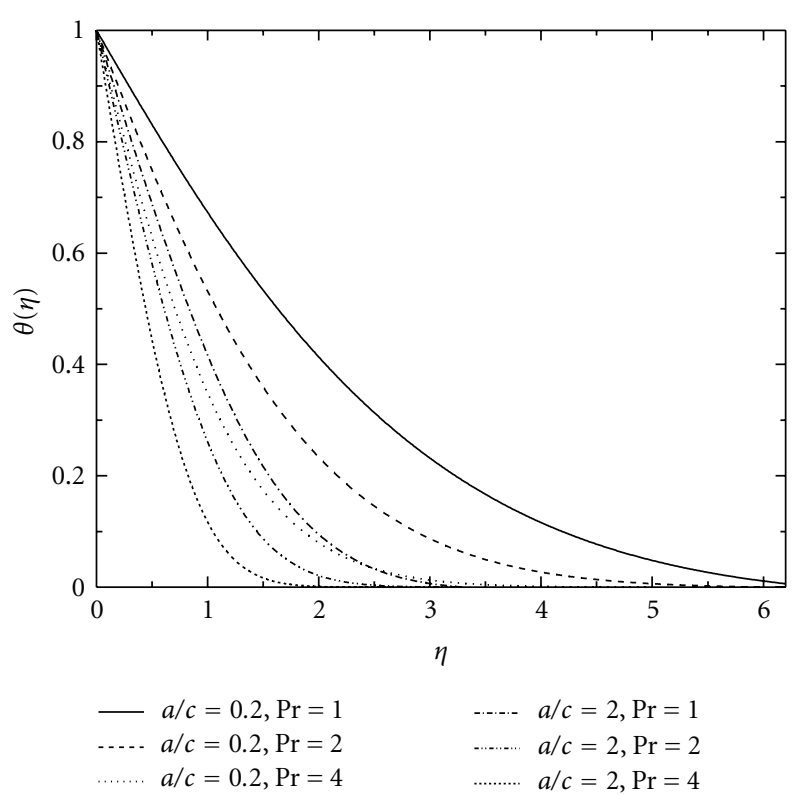

Figure 6: Variation of $\theta(\eta)$ with $\eta$ for different values of $\operatorname{Pr}$ and $a / c$ with $f_{w}=0, n=0.8, M=0.5$, and $N_{R}=1.0$.

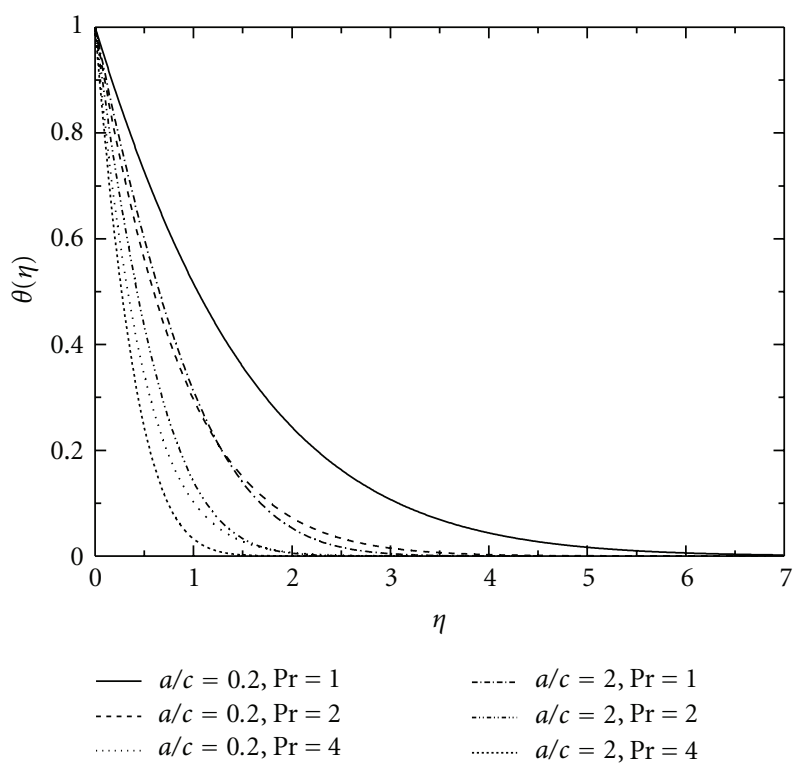

FIgURE 7: Variation of $\theta(\eta)$ with $\eta$ for different values of $\operatorname{Pr}$ and $a / c$ with $f_{w}=1, n=0.8, M=0.5$, and $N_{R}=1.0$.

divergence of the radiation heat flux $\partial q_{r} / \partial y$ decreases with increases in the Rosseland radiative absorption coefficient $K^{*}$ increases (see (16)) which in turn decreases the rate of radiative heat transfer to the fluid which causes the fluid temperature to decrease. Figures 11-13 also depict that the temperature at a given point decreases with increasing $a / c$ for the fixed values of $M, n, \operatorname{Pr}$, and $N_{R}$. Figures 11-13 also depict that the temperature at a given point decreases with increasing $a / c$ for the fixed values of $M, n, \mathrm{Pr}$, and $N_{R}$. 


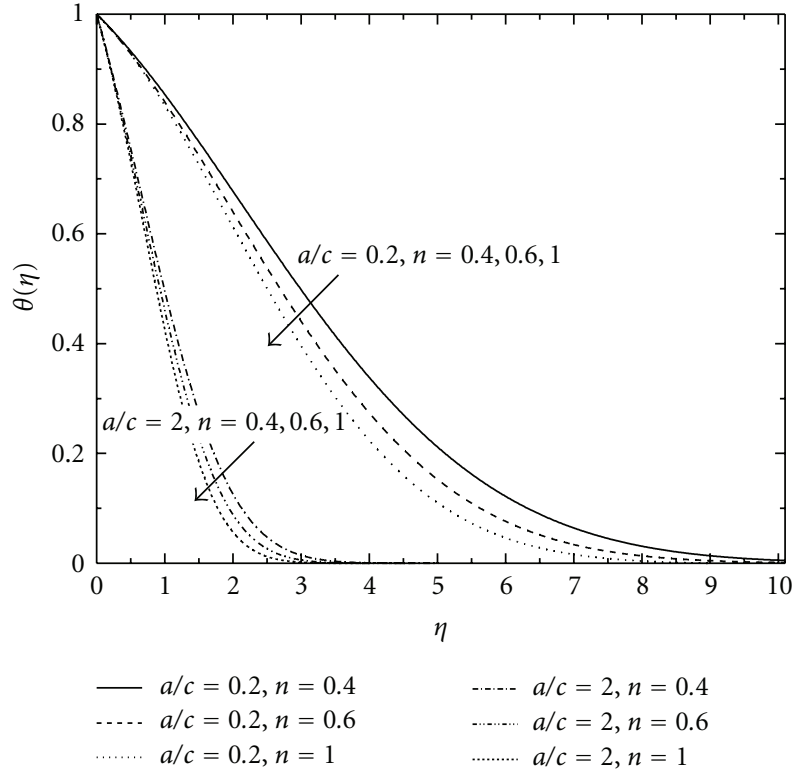

Figure 8: Variation of $\theta(\eta)$ with $\eta$ for different values of $n$ and $a / c$ with $f_{w}=-1, M=1.0, \operatorname{Pr}=2.0$, and $N_{R}=1.0$.

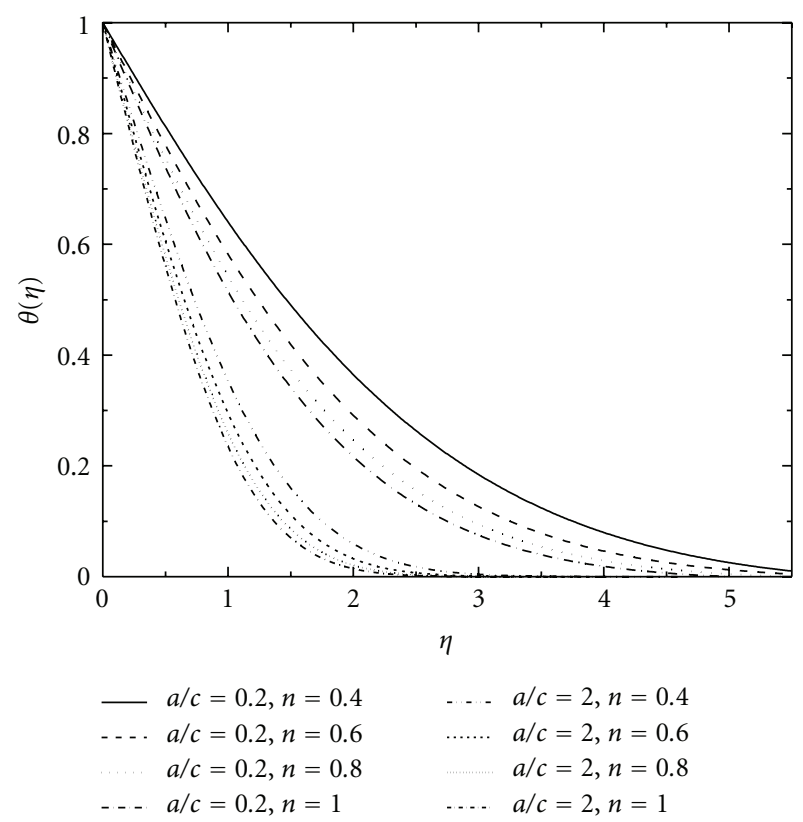

Figure 9: Variation of $\theta(\eta)$ with $\eta$ for different values of $n$ and $a / c$ with $f_{w}=0, M=1.0, \operatorname{Pr}=2.0$, and $N_{R}=1.0$.

Figure 14 shows that the temperature at a given point increases with increase in $M$ when $a / c<1(=0.2)$ for fixed values of $n, \mathrm{Pr}$, and $N_{R}$. This is due to the fact that as the strength of magnetic field $M$ increases the Lorentz force which opposes the flow in the boundary layer that is responsible for increase of the temperature in the thermal boundary layer. Further, it is observed from this figure that the temperature at a given point decreases with increase in $M$ when $a / c>1$ for fixed values of $n, \operatorname{Pr}$, and $N_{R}$. This

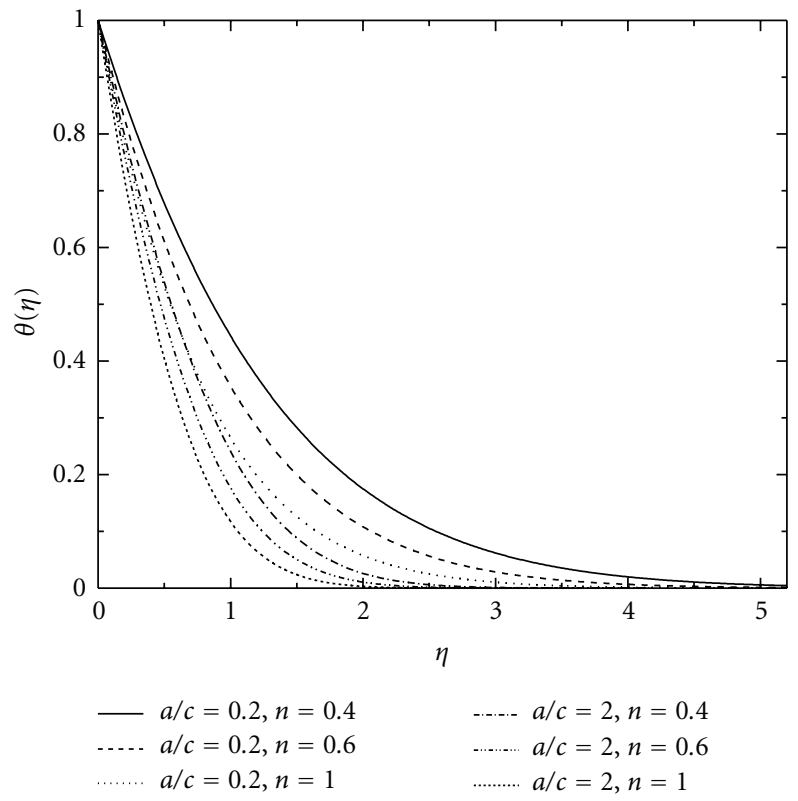

Figure 10: Variation of $\theta(\eta)$ with $\eta$ for different values of $n$ and $a / c$ with $f_{w}=1, M=1.0, \operatorname{Pr}=2.0$, and $N_{R}=1.0$.

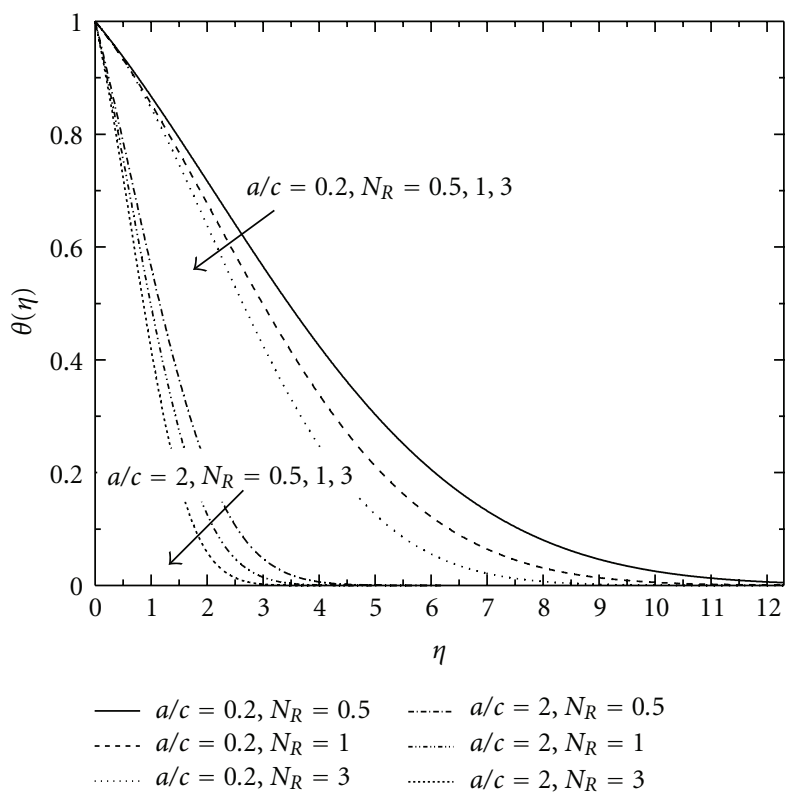

Figure 11: Variation of $\theta(\eta)$ with $\eta$ for different values of $N_{R}$ and $a / c$ with $f_{w}=-1, n=0.4, M=1.0$, and $\operatorname{Pr}=2.0$.

paradoxical result can be explained by the fact that when $a / c<1$, the velocity of the stretching sheet exceeds the velocity of the inviscid stream and an inverted boundary layer is formed near the surface. Thus one would expect that the velocity at a point in this boundary layer increases with increase in $M$ and thereby decreases the temperature in the thermal boundary layer. 


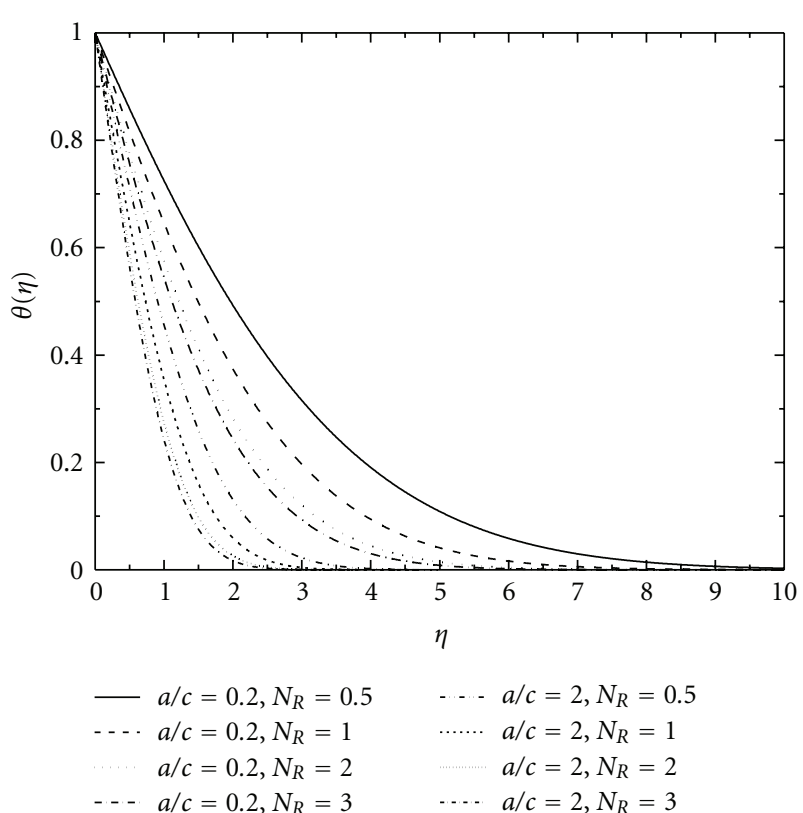

Figure 12: Variation of $\theta(\eta)$ with $\eta$ for different values of $N_{R}$ and $a / c$ with $f_{w}=0, n=0.4, M=1.0$, and $\operatorname{Pr}=2.0$.

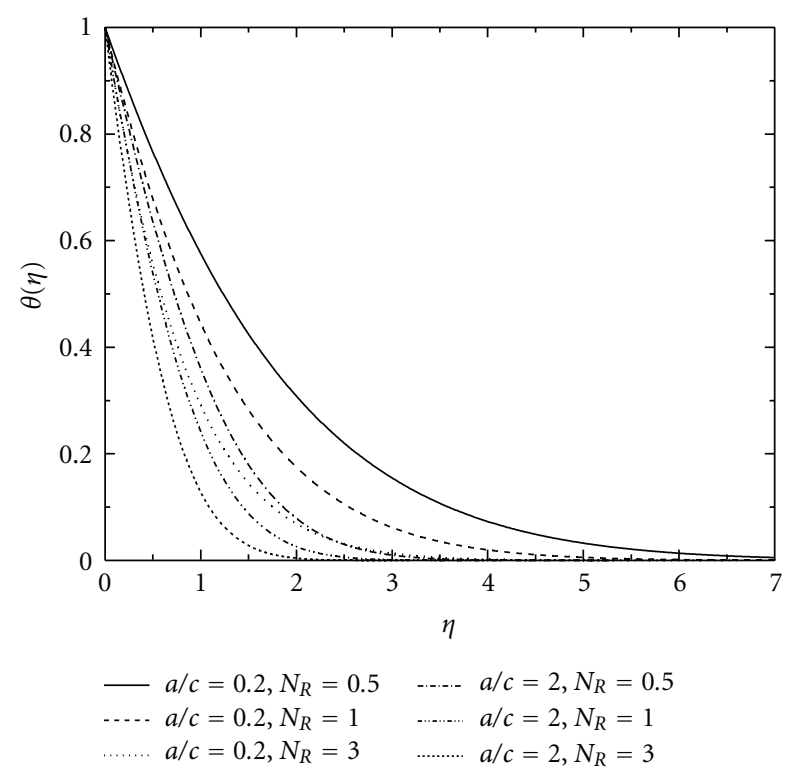

Figure 13: Variation of $\theta(\eta)$ with $\eta$ for different values of $N_{R}$ and $a / c$ with $f_{w}=1, n=0.4, M=1.0$, and $\operatorname{Pr}=2.0$.

\section{Conclusions}

The present paper deals with the analysis of the heat transfer in the steady two-dimensional MHD stagnation-point flow of an electrically conducting power-law fluid over a stretching surface. The sheet is stretched in its plane with a velocity $c x, x$ being the distance along the plate from the stagnation point. The following conclusions can be drawn from the numerical results obtained.

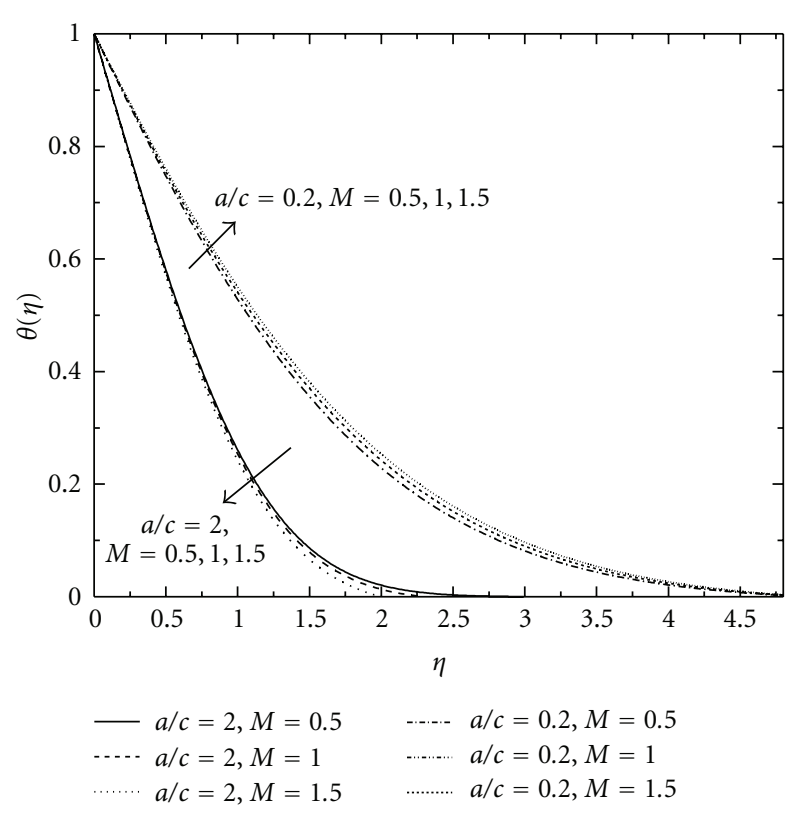

Figure 14: Variation of $\theta(\eta)$ with $\eta$ for different values of $M$ and $a / c$ with $f_{w}=0, n=0.8, \operatorname{Pr}=2.0$, and $N_{R}=1.0$.

(i) When $a / c<1$, the velocity at a point in a neighbourhood of the stretching plate increases with increase in the power-law index but velocity at a point decreases with the increase in power-law index, beyond certain distance from stretching sheet, whereas opposite trend is observed when $a / c>1$. Further it is found that when $f_{w}<1$, then the velocity profile increases for $a / c<1$, whereas reverse effect is found when $a / c>1$. But for the case when $f_{w}>1$, then the velocity profile decreases for $a / c<1$, whereas reverse effect is found when $a / c>1$.

(ii) Temperature decreases with increase in power-law index for both $a / c<1$ and $a / c>1$.

(iii) Temperature increases with increase in the magnetic parameter when $a / c<1$ and decreases when $a / c>1$.

(iv) Temperature decreases with increase in the value of the thermal radiation parameter for both $a / c<1$ and $a / c>1$.

(v) Temperature decreases with increase in the value of the Prandtl number for both $a / c<1$ and $a / c>1$.

\section{Acknowledgment}

The work of two of the authors (T. R. Mahapatra and D. Pal) is supported under SAP (DRS PHASE II) program of UGC, New Delhi, India. The authors wish to thank the referees for helpful comments and suggestions which improved the quality of the paper considerably. 


\section{References}

[1] Y. G. Sapunkov, "Self-similarity solutions of non-Newtonian fluid boundary in MHD," Mekhanika Zhidkosti I Gaza, vol. 2, pp. 77-82, 1967.

[2] L. J. Crane, "Flow past a stretching plate," Zeitschrift für Angewandte Mathematik und Physik, vol. 21, no. 4, pp. 645-647, 1970.

[3] K. B. Pavlov, "Magnetohydrodynamic flow of an incompressible viscous fluid caused by the deformation of plane surface," Magnetohydrodynamics, vol. 4, pp. 645-647, 1974.

[4] L. K. Martinson and K. B. Pavlov, "Magnetohydrodynamics on non-Newtonian fluids," Magnetohydrodynamics, vol. 11, no. 1, p. 59, 1975.

[5] P. S. Gupta and A. S. Gupta, "Heat and mass transfer on a stretching sheet with suction or blowing," The Canadian Journal of Chemical Engineering, vol. 55, pp. 744-746, 1977.

[6] P. Carragher and L. J. Crane, "Heat transfer on a continuous stretching sheet," Zeitschrift für Angewandte Mathematik und Mechanik, vol. 62, p. 564, 1982.

[7] M. Massoudi and M. Ramezan, "Boundary layers heat transfer analysis of a viscoelastic fluid at a stagnation point," ASME Heat Transfer Division, vol. 130, pp. 81-86, 1990.

[8] M. Massoudi and M. Ramezan, "Heat transfer analysis of a viscoelastic fluid at a stagnation point," Mechanics Research Communications, vol. 19, no. 2, pp. 129-134, 1992.

[9] H. I. Andersson, K. H. Bech, and B. S. Dandapat, "Magnetohydrodynamic flow of a power-law fluid over a stretching sheet," International Journal of Non-Linear Mechanics, vol. 27, no. 6, pp. 929-936, 1992.

[10] V. K. Garg, "Heat transfer due to stagnation point flow of a non-Newtonian fluid," Acta Mechanica, vol. 104, no. 3-4, pp. 159-171, 1994.

[11] T. R. Mahapatra and A. S. Gupta, "Magnetohydrodynamic stagnation-point flow towards a stretching sheet," Acta Mechanica, vol. 152, no. 1-4, pp. 191-196, 2001.

[12] T. R. Mahapatra and A. S. Gupta, "Heat transfer in stagnationpoint flow towards a stretching sheet," Heat and Mass Transfer, vol. 38, no. 6, pp. 517-521, 2002.

[13] S. J. Liao and I. Pop, "Explicit analytic solution for similarity boundary layer equations," International Journal of Heat and Mass Transfer, vol. 47, no. 1, pp. 75-85, 2004.

[14] R. Nazar, N. Amin, and I. Pop, "Unsteady boundary layer flow due to a stretching surface in a rotating fluid," Mechanics Research Communications, vol. 31, no. 1, pp. 121-128, 2004.

[15] K. V. Prasad, D. Pal, and P. S. Datti, "MHD power-law fluid flow and heat transfer over a non-isothermal stretching sheet," Communications in Nonlinear Science and Numerical Simulation, vol. 14, no. 5, pp. 2178-2189, 2009.

[16] T. R. Mahapatra, S. K. Nandy, and A. S. Gupta, "Magnetohydrodynamic stagnation-point flow of a power-law fluid towards a stretching surface," International Journal of NonLinear Mechanics, vol. 44, no. 2, pp. 123-128, 2009.

[17] M. A. Hossain and H. S. Takhar, "Radiation effect on mixed convection along a vertical plate with uniform surface temperature," Heat and Mass Transfer, vol. 31, no. 4, pp. 243-248, 1996.

[18] D. Pal, "Heat and mass transfer in stagnation-point flow towards a stretching surface in the presence of buoyancy force and thermal radiation," Meccanica, vol. 44, no. 2, pp. 145-158, 2009.

[19] J. A. Shercliff, A Textbook of Magnetohydrodynamic, Pergamon Press, Oxford, UK, 1965.
[20] A. Raptis, "Radiation and free convection flow through a porous medium," International Communications in Heat and Mass Transfer, vol. 25, no. 2, pp. 289-295, 1998. 

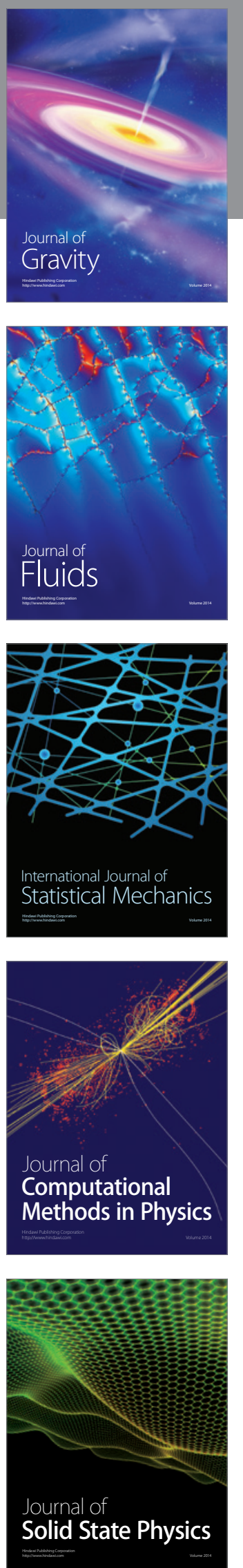
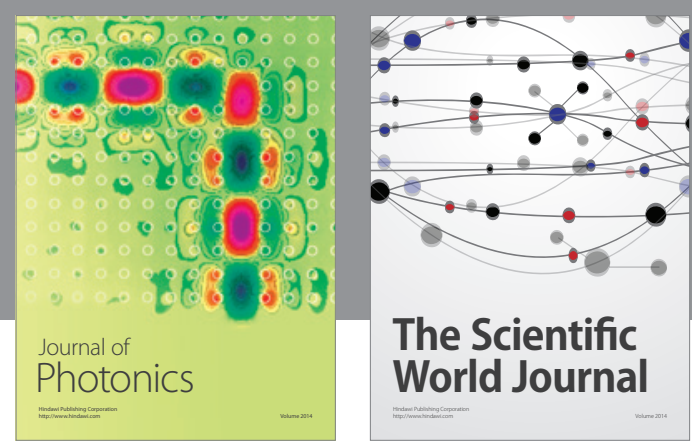

The Scientific World Journal

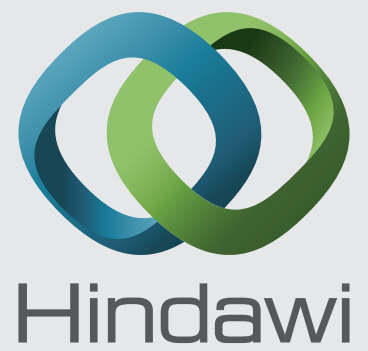

Submit your manuscripts at http://www.hindawi.com
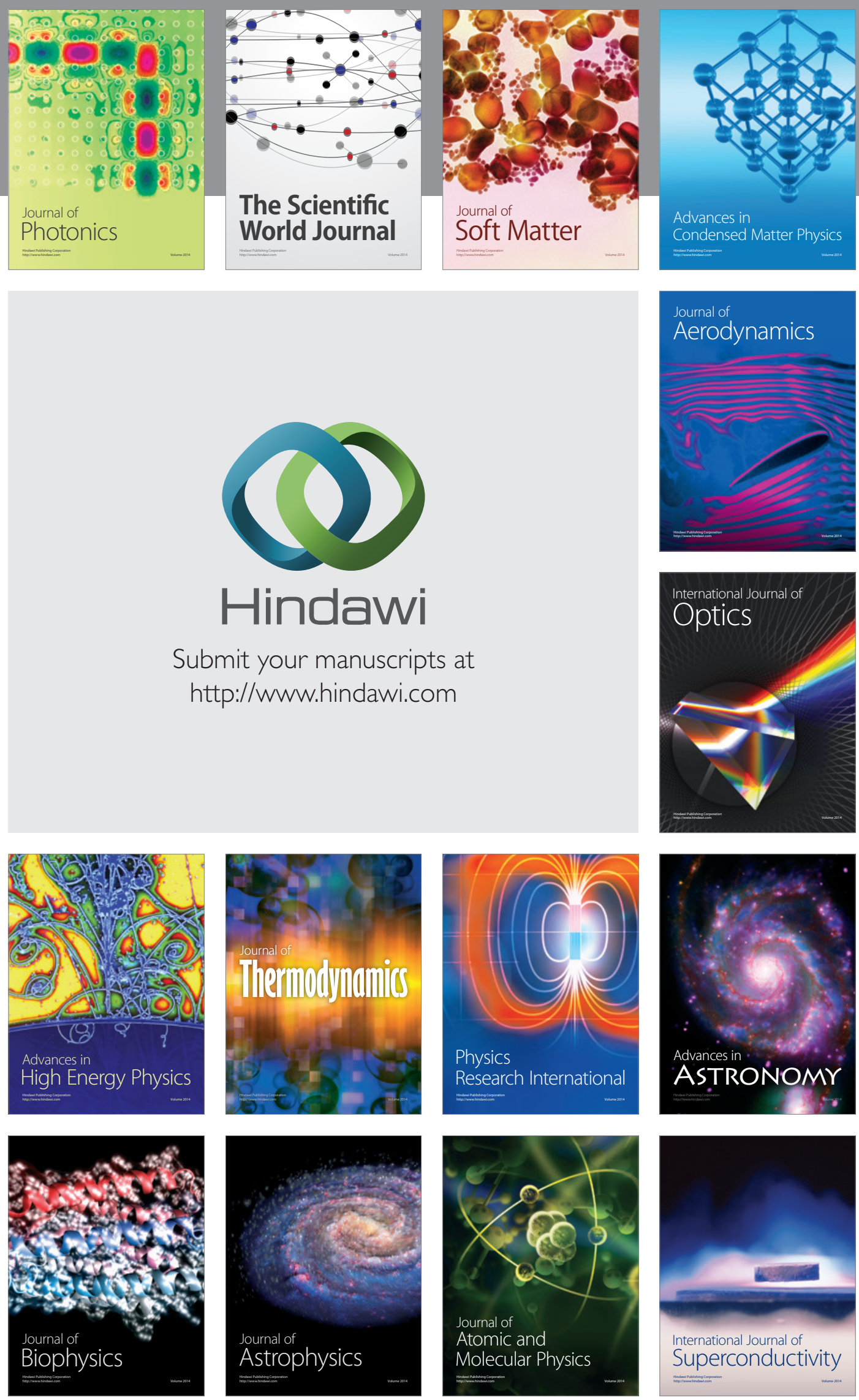
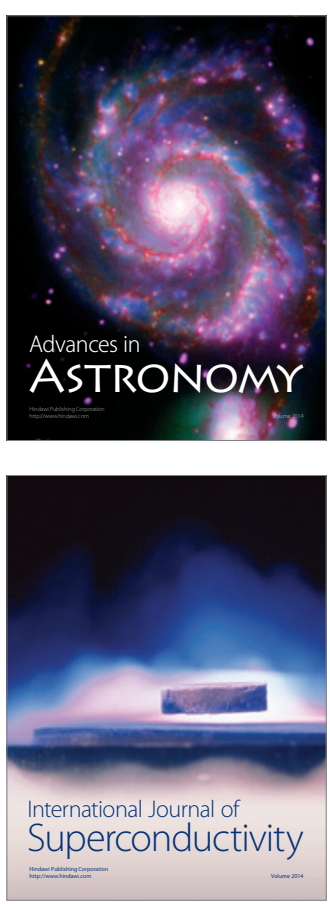